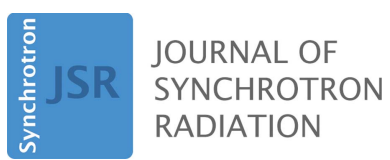

ISSN $1600-5775$

Received 15 August 2019

Accepted 27 March 2020

Edited by G. Grübel, HASYLAB at DESY, Germany

‡ On leave from Karlsruhe Institute of Technology, PO Box 3640, D-76021 Karlsruhe, Germany.

Keywords: superradiant emission; terahertz radiation; X-ray free-electron laser; ultrafast phenomena; terahertz control.

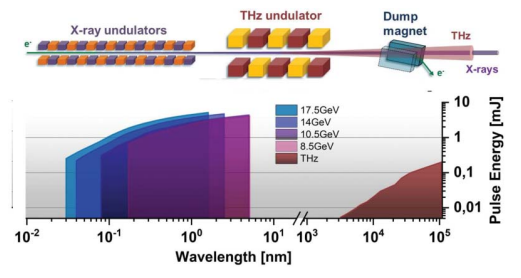

OPEN $\odot$ ACCESS

\title{
Volt-per-Ångstrom terahertz fields from X-ray free-electron lasers
}

T. Tanikawa, ${ }^{a}$ S. Karabekyan, ${ }^{\text {a }}$ S. Kovalev, ${ }^{\text {b }}$ S. Casalbuoni, ${ }^{a} \neq$ V. Asgekar, ${ }^{c}$

S. Bonetti, ${ }^{\text {d,e }}$ S. Wall, ${ }^{\text {f }}$ T. Laarmann, ${ }^{\text {, h }}$ D. Turchinovich, ${ }^{\text {i }}$ P. Zalden, ${ }^{\text {a }}$

T. Kampfrath, ${ }^{j}$ A. S. Fisher, ${ }^{k}$ N. Stojanovic, ${ }^{\mathrm{g}, \mathrm{I}}$ M. Gensch ${ }^{\mathrm{l}, \mathrm{m} *}$ and G. Geloni ${ }^{\mathrm{a} *}$

aEuropean XFEL, Holzkoppel 4, 22869 Schenefeld, Germany, ${ }^{\mathbf{b}}$ Helmholtz-Zentrum Dresden-Rossendorf, Bautzner Landstraße 400, 01328 Dresden, Germany, ' Physics Department, S. P. Pune University, Pune 411 007, India, ${ }^{\mathbf{d} D e p a r t m e n t}$ of Physics, Stockholm University, 10691 Stockholm, Sweden, ${ }^{\mathbf{e}}$ Department of Molecular Sciences and Nanosystems, Ca' Foscari University of Venice, 30172 Venice, Italy, ' ICFO, Avinguda Carl Friedrich Gauss 3, 08860 Castelldefels, Barcelona, Spain, ${ }^{\mathbf{g}}$ Deutsches Elektronen Synchrotron DESY, Notkestraße 85, 22607 Hamburg, Germany, h'The Hamburg Centre for Ultrafast Imaging CUI, Luruper Chaussee 149, 22761 Hamburg, Germany, 'Fakultät für Physik, Universität Bielefeld, Universitätsstraße 25, 33615 Bielefeld, Germany, 'ंDepartment of Physics, Freie Universität Berlin, Arnimallee 14, 14195 Berlin, Germany, kSLAC National Accelerator Laboratory, 2575 Sand Hill Road, Menlo Park, CA 94025, USA, 'DLR - Institute for Optical Sensor Systems, Rutherfordstraße 2, 12489 Berlin, Germany, and m Institute of Optics and Atomic Physics, Technische Universität Berlin, Straße des 17 Juni 135, 10623 Berlin, Germany. *Correspondence e-mail: michael.gensch@dlr.de, gianluca.geloni@xfel.eu

The electron linear accelerators driving modern X-ray free-electron lasers can emit intense, tunable, quasi-monochromatic terahertz $(\mathrm{THz})$ transients with peak electric fields of $\mathrm{V}^{-1}$ and peak magnetic fields in excess of $10 \mathrm{~T}$ when a purpose-built, compact, superconducting $\mathrm{THz}$ undulator is implemented. New research avenues such as X-ray movies of THz-driven mode-selective chemistry come into reach by making dual use of the ultra-short $\mathrm{GeV}$ electron bunches, possible by a rather minor extension of the infrastructure.

X-ray free-electron lasers [XFELS; see, for example, Pellegrini (2016) for a recent review] are currently the brightest, tunable sources of short X-ray pulses available for basic scientific research. Various techniques based on X-ray scattering, X-ray diffraction, and X-ray spectroscopies enable element-specific probing of dynamical processes in materials on the timescales from femtoseconds to nanoseconds and/or with a spatial resolution down to the Ångstrom level (Dunne, 2018; Marx, 2017; Fromme, 2015). As outlined in this Short communication, the kilometre-long linear accelerators (linacs) driving these facilities are also capable of producing narrowband, frequency-tunable terahertz $(\mathrm{THz})$ transients with $\mathrm{V} \AA^{-1}$-level THz fields.

The THz generation is based on superradiant emission from $\mathrm{GeV}$-scale electron bunches in a specifically designed $\mathrm{THz}$ undulator positioned between the X-ray undulators and the electron beam dump (see Fig. 1). A first prototype of such a scheme is operational in the electron linac of the free-electron laser FLASH (Gensch et al., 2008) and enables one to probe THz-driven processes in the XUV spectral range. However, implementation into the kilometre-long, GeV-level linacs of hard X-ray FELs was until recently considered unfeasible because of two major technological obstacles. Firstly, THz undulators based on conventional technology and sufficiently large gap would require an unreasonably long period length of $10 \mathrm{~m}$ or more. Secondly, the required beam transport over a few hundreds of meters would lead to unrealistic scenarios 


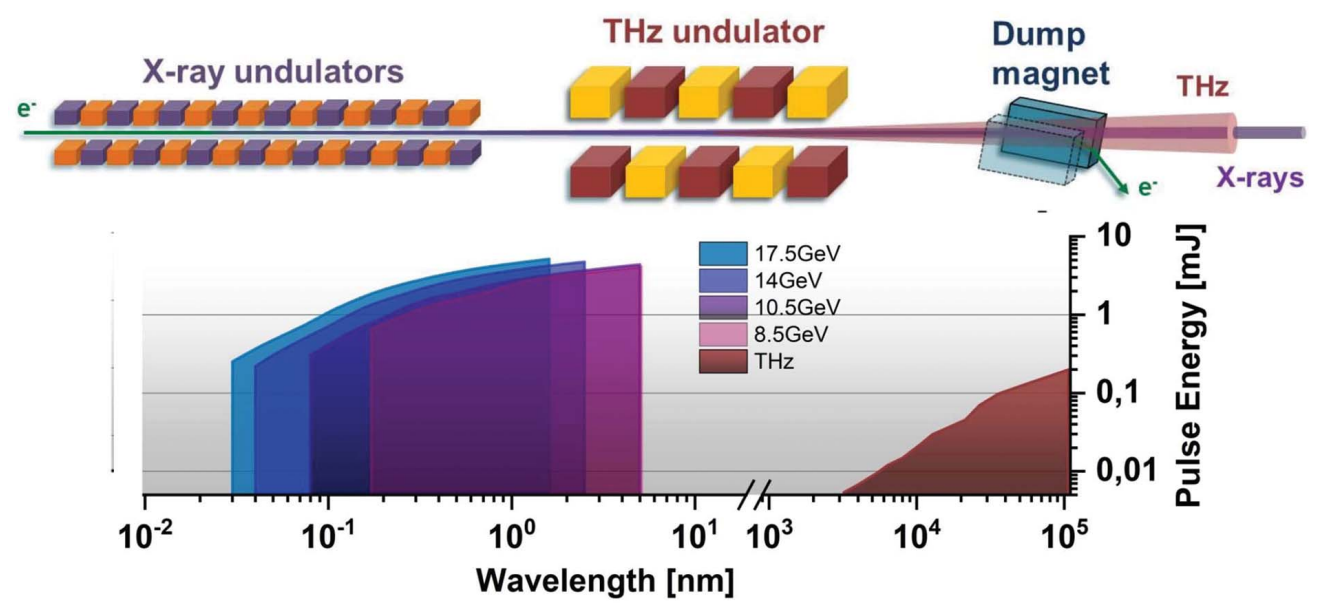

Figure 1

Principal scheme (top) and expected pulse energies from the X-ray to THz regime (bottom) for the example of the European XFEL. Relativistic GeV ultra-short and highly charged electron bunches first generate X-ray pulses by self-amplified spontaneous emission (SASE) in soft and hard X-ray undulators in the few $\mathrm{mJ}$ regime (Pellegrini, 2016). After passing the X-ray undulator section the electron bunches enter an additional few-period THz undulator and generate tunable, narrowband THz pulses with up to few $100 \mu \mathrm{J}$ pulse energy by superradiant emission (Tanikawa et al., 2019). X-ray pulse energies are calculated at saturation for electron beam energies of 8.5, 10.5, 14 and $17.5 \mathrm{GeV}$ and a bunch charge of $500 \mathrm{pC}$ (Schneidmiller \& Yurkov, 2017). (Note that when a certain peak current is fixed, the X-ray pulse duration, and therefore its energy, will scale roughly linearly with the charge. Deviations mainly occur due to the dependence of the electron beam parameters on the bunch charge. Currently, in operations electron energies of $11.5,14$ and $16.5 \mathrm{GeV}$ with a charge of $250 \mathrm{pC}$ are routinely used.)

such as complex all-optical X-ray delay lines. As we show here, several technological breakthroughs of recent years allow meanwhile to overcome these technological hurdles.

Taking the European XFEL with its particularly high beam energy as an example, we showed the feasibility of a compact eight-period undulator with a period length of $1 \mathrm{~m}$, an on-axis peak field of $7.3 \mathrm{~T}$ and a magnetic gap of $50 \mathrm{~mm}$, using NbTibased superconducting undulator technology (Casalbuoni $e t$ al., 2018; Tanikawa et al., 2019). The device has an overall magnetic length of $8 \mathrm{~m}$ and is hence by an order of magnitude shorter than normal conducting undulators based on copper coils or permanent magnets to produce the same frequency tuning range between 3 and $100 \mathrm{THz}$ for the first harmonic. ${ }^{1}$ The second obstacle, the significant delay of few tens of nanoseconds in the time of arrival of X-ray and $\mathrm{THz}$ pulses from the same electron bunch, can be overcome by recently successfully demonstrated double-bunch schemes (Zapolnova et al., 2018). In combination with the recent advances in pulseresolved detection and arrival-time measurements (see, for example, Kovalev et al., 2017; Bionta et al., 2014), THz and $\mathrm{X}$-ray pulses can now be provided with a few femtoseconds timing precision despite independent transport beamlines of several $100 \mathrm{~m}$ (see Tanikawa et al., 2019, and references therein). Utilizing electron bunch forms calculated for the European XFEL allows one to predict the achievable pulse energies and electric and magnetic peak fields (Tanikawa $e t$ al., 2019).

As shown in Fig. 2, values of $>0.2 \mathrm{~V}^{-1}$ will be reached in a wide range of the emitted $\mathrm{THz}$ frequencies between 8 and $100 \mathrm{THz}$. A field strength of $0.5 \mathrm{~V} \AA^{-1}$ can be achieved in a

\footnotetext{
${ }^{\mathbf{1}}$ Note that a large gap is needed in the $\mathrm{THz}$ frequency range to reduce the frequency cut-off of the vacuum chamber, and to transmit most of the $\mathrm{THz}$ radiation of interest, starting from the first harmonic up to higher frequencies.
}

large part of the so-called molecular fingerprint region between 15 and $100 \mathrm{THz}$, which contains the vibrational and vibrational-rotational modes of highly relevant chemical bonds (e.g. $\mathrm{C}-\mathrm{H}_{x}, \mathrm{C}-\mathrm{O}, \mathrm{C}=\mathrm{O}, \mathrm{NO}_{2}, \mathrm{NH}_{2}$ ), thereby opening up the opportunity to observe mode-selective chemistry in molecules and clusters (Zewail, 1980; Lee et al., 2012) in an $\mathrm{X}$-ray molecular movie for the first time. Of fundamental relevance is the opportunity to investigate the molecular dynamics of water in the $\mathrm{THz}$ regime, where $\mathrm{V}^{-1}$ level $\mathrm{THz}$ pulses can induce structural non-equilibrium states, also extending across the recently discovered liquid-liquid phase transition (Kim et al., 2017). In solids, the provided $\mathrm{THz}$ frequency range covers the stretching and bending phonon modes of many metal-ion-oxygen bonds (e.g. $\mathrm{Mn}-\mathrm{O}, \mathrm{Cu}-\mathrm{O}$, $\mathrm{Fe}-\mathrm{O}$ ), which typically lie between 8 and $20 \mathrm{THz}$. When driven by sufficiently strong $\mathrm{THz}$ fields, these modes have been recently shown to play an important role in the control of transient electronic and magnetic phases in correlated quantum systems (Buzzi et al., 2018). Further, the achievable peak $\mathrm{THz}$ magnetic fields are in the few-Tesla range over the whole frequency spectrum from 3 to $100 \mathrm{THz}$. These field magnitudes are sufficient for selective excitation of magnetic resonances far into the nonlinear regime (Kampfrath et al., 2013), where the fastest predicted magnetization reversal mechanism (Tudosa et al., 2004), i.e. coherent magnetization reversal on a $\sim 1 \mathrm{ps}$ timescale, is expected to occur. Combined with powerful $\mathrm{THz}$ streaking techniques, the $\mathrm{V} \AA^{-1} \mathrm{THz}$ electric fields allow to unravel short-wavelength FEL-induced correlated electron and nuclear dynamics in atoms, molecules, and nanoparticles on extreme time scales (Oelze et al., 2019). Finally, important emerging technologies such as $\mathrm{THz}$ lightwave electronics (Reimann et al., 2018) will benefit from possibilities to probe strong-field $\mathrm{THz}$-driven dynamics in solid systems with a nontrivial bulk electronic structure by 

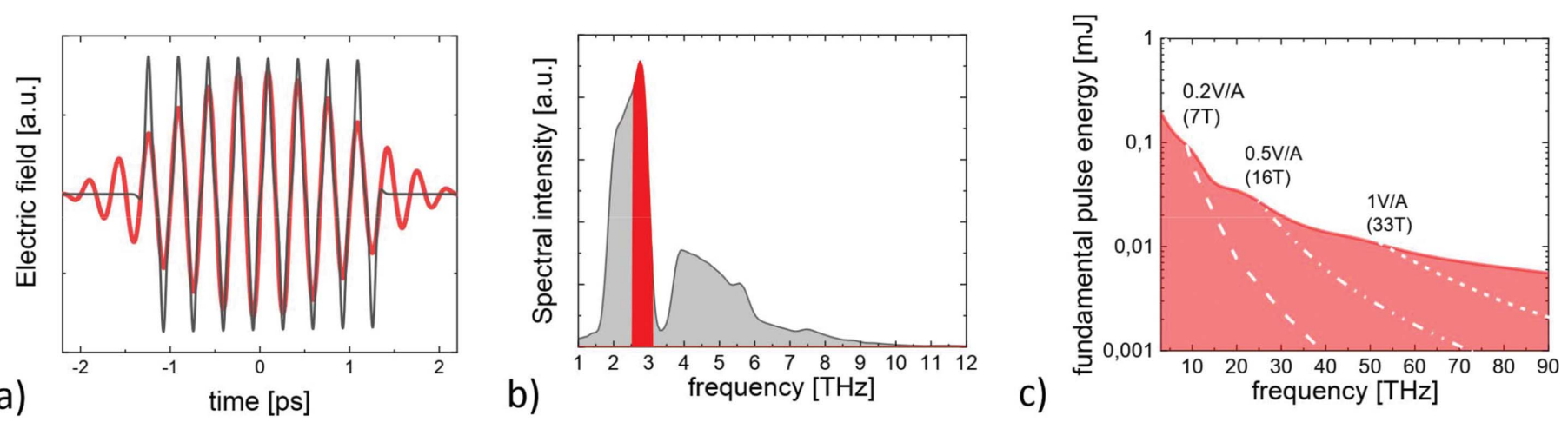

Figure 2

$\mathrm{THz}$ waveforms emitted by the THz undulator for a central frequency of $\sim 3 \mathrm{THz}(a)$ and corresponding $\mathrm{THz}$ spectrum $(b)$. Data are shown for the total emitted THz spectrum (black) and for a bandpass filter of $20 \%$ around the fundamental frequency (red). The pulse energies as emitted into a bandwidth of $20 \%$ around the fundamental are shown in $(c)$. The waveforms are derived from an analytical calculation [described in detail by Tanikawa et al. (2019)] assuming the most optimal bunch charge and corresponding bunch form $(<5.8 \mathrm{THz} / 500 \mathrm{pC},<15 \mathrm{THz} / 250 \mathrm{pC},<75 \mathrm{THz} / 100 \mathrm{pC}$ and $20 \mathrm{pC}$ at higher frequencies). The achievable peak THz fields, assuming a moderate numerical aperture of 0.25 , are indicated in $(c)$ by dashed lines. Note that one does not expect to observe significant changes in the electron bunch form for beam energies between 8.5 and $17.5 \mathrm{GeV}$ and hence the THz pulse energies are the same.

techniques such as hard X-ray angle-resolved photoemission spectroscopy. In summary, a modest extension by additional superconducting undulators and $\mathrm{THz}$ beamlines enables additional use of the $\mathrm{GeV}$-scale electron beams in modern XFELs for the generation of $\mathrm{V} \AA^{-1}$-level THz fields. Thereby $\mathrm{X}$-ray movies on femtosecond time and Ångstrom length scales of long-debated phenomena become feasible, opening up completely new research avenues.

\section{Funding information}

VA acknowledges support from the Alexander von Humboldt Foundation, Germany. DT acknowledges the project 'Nonequilibrium dynamics in solids probed by terahertz fields' funded by the Deutsche Forschungsgemeinschaft (DFG, German Research Foundation) - project number 278162697 SFB 1242. TK acknowledges funding through the collaborative research center TRR 227 'Ultrafast spin dynamics' (project A05). SW was supported by European Union's Horizon 2020 research and innovation programme (Grant Agreement No. 758461), Spanish MINECO (Severo Ochoa grant SEV-2015-0522, SEV2015-0496) as well as Fundació Privada Cellex, and CERCA Programme/Generalitat de Catalunya.

\section{References}

Bionta, M. R., Hartmann, N., Weaver, M., French, D., Nicholson, D. J., Cryan, J. P., Glownia, J. M., Baker, K., Bostedt, C., Chollet, M., Ding, Y., Fritz, D. M., Fry, A. R., Kane, D. J., Krzywinski, J., Lemke, H. T., Messerschmidt, M., Schorb, S., Zhu, D., White, W. E. \& Coffee, R. N. (2014). Rev. Sci. Instrum. 85, 083116.

Buzzi, M., Först, M., Mankowsky, R. \& Cavalleri, A. (2018). Nat. Rev. Mater. 3, 299-311.
Casalbuoni, S., Glamann, N., Grau, A., Holubek, T., Saez de Jauregui, D., Bauer, S., Boffo, C., Gerhard, T., Turenne, M. \& Walter, W. (2018). Synchrotron Radiat. News, 31(3), 24-28.

Dunne, M. (2018). Nat. Rev. Mater. 3, 290-292.

Fromme, P. (2015). Nat. Chem. Biol. 11, 895-899.

Gensch, M., Bittner, L., Chesnov, A., Delsim-Hashemi, H., Drescher, M., Faatz, B., Feldhaus, J., Fruehling, U., Geloni, G. A., Gerth, Ch., Grimm, O., Hahn, U., Hesse, M., Kapitzki, S., Kocharyan, V., Kozlov, O., Matyushevsky, E., Morozov, N., Petrov, D., Ploenjes, E., Roehling, M., Rossbach, J., Saldin, E. L., Schmidt, B., Schmueser, P., Schneidmiller, E. A., Syresin, E., Willner, A. \& Yurkov, M. V. (2008). Infrared Phys. Technol. 51, 423-425.

Kampfrath, T., Tanaka, K. \& Nelson, K. A. (2013). Nat. Photon. 7, 680-690.

Kim, K. H., Späh, A., Pathak, H., Perakis, F., Mariedahl, D., AmannWinkel, K., Sellberg, J. A., Lee, J. H., Kim, S., Park, J., Nam, K. H., Katayama, T. \& Nilsson, A. (2017). Science, 358, 1589-1593.

Kovalev, S., Green, B., Golz, T., Maehrlein, S., Stojanovic, N., Fisher, A. S., Kampfrath, T. \& Gensch, M. (2017). Struct. Dyn. 4, 024301.

Lee, J., Suits, A. G., Schlegel, H. B. \& Li, W. (2012). J. Phys. Chem. Lett. 3, 2541-2547.

Marx, V. (2017). Nat. Methods, 14, 843-846.

Oelze, T., Schütte, B., Müller, J. P., Müller, M., Wieland, M., Frühling, U., Drescher, M., Golz, T., Al-Shemmary, A., Stojanovic, N. \& Krikunova, M. (2019). Phys. Rev. A, 99, 043423.

Pellegrini, C. (2016). Phys. Scr. T169, 014004.

Reimann, J., Schlauderer, S., Schmid, C. P., Langer, F., Baierl, S., Kokh, K. A., Tereshchenko, O. E., Kimura, A., Lange, C., Güdde, J., Höfer, U. \& Huber, R. (2018). Nature, 562, 396-400.

Schneidmiller, E. A. \& Yurkov, M. V. (2017). Proceedings of the 38th International Free Electron Laser Conference (FEL2017), 20-25 August 2017, Santa Fe, NM, USA. pp. 109-112. MOP033.

Tanikawa, T., Karabekyan, S., Kovalev, S., Casalbuoni, S., Asgekar, V., Serkez, S., Geloni, G. \& Gensch, M. (2019). J. Instrum. 14, P05024.

Tudosa, I., Stamm, C., Kashuba, A. B., King, F., Siegmann, H. C., Stöhr, J., Ju, G., Lu, B. \& Weller, D. (2004). Nature, 428, 831-833.

Zapolnova, E., Golz, T., Pan, R., Klose, K., Schreiber, S. \& Stojanovic, N. (2018). J. Synchrotron Rad. 25, 39-43.

Zewail, A. H. (1980). Phys. Today, 33, 27-33. 